\title{
MODELAGEM DA GASEIFICAÇÃO DA BIOMASSA EM REATOR DE LEITO FLUIDIZADO
}

\author{
G. P. MIRANDA ${ }^{1}$, P. A. MELO ${ }^{1}$ e J. C. C. S. PINTO ${ }^{1}$ \\ ${ }^{1}$ Universidade Federal do Rio de Janeiro, COPPE, Programa de Engenharia Química \\ E-mail para contato: gillimiranda@yahoo.com.br
}

\begin{abstract}
RESUMO - A obtenção de energia por meio do processo de gaseificação da biomassa é favorecida com o uso de reatores de leito fluidizado, por possibilitarem eficiente transferência de massa e calor entre as fases. Estudos sobre a modelagem da gaseificação nestes reatores mostram discrepâncias com dados experimentais, o que pode ser explicado pela complexidade deste sistema reacional físico-químico. Para descrever o processo de forma mais eficaz, um modelo fluidodinâmico, não-estacionário, foi proposto, constituído por: i) um modelo cinético representando a biomassa como um composto constituído por carbono, hidrogênio e oxigênio $\left(\mathrm{C}_{\mathrm{x}} \mathrm{H}_{\mathrm{y}} \mathrm{O}_{\mathrm{z}}\right)$; ii) um modelo fluidodinâmico para descrever o comportamento do leito fluidizado; iii) balanços de massa e de energia, escritos para as fases bolha, emulsão e sólida. Os resultados obtidos mostram que uma biomassa real pode induzir comportamento muito distinto do reator, modificando expressivamente as concentrações de produtos na saída e o comportamento dinâmico do processo. Assim, a implementação de um modelo cinético para representar uma biomassa mais adequada possibilita a obtenção de resultados mais coerentes com a realidade de operação industrial, especificamente, com maior taxa de formação de gás de síntese $\left(\mathrm{H}_{2} \mathrm{e} \mathrm{CO}\right)$, que é essencial para a indústria química.
\end{abstract}

\section{INTRODUÇÃO}

Na tentativa de minimizar os impactos causados pela emissão de gases tóxicos na atmosfera provenientes da queima de combustíveis fósseis, medidas têm sido propostas a fim de reduzir o uso destes combustíveis e buscar soluções de cunho renovável. Dentre as opções já conhecidas de fontes renováveis de energia, a biomassa se destaca no Brasil, pois a sua vasta área territorial fornece condições ótimas para a produção da matéria-prima necessária ao processo (MIRANDA, 2014).

Energia pode ser obtida a partir da biomassa principalmente por processos bioquímicos (por exemplo, fermentação e digestão anaeróbica), e processos termoquímicos, que fazem uso de calor, pressão e catalisadores (por exemplo, pirólise e gaseificação) (AHMED et al., 2012). A gaseificação é uma oxidação parcial conduzida a elevadas temperaturas e, apesar de constituir um processo bastante complexo, há grande interesse industrial neste processo porque ele permite a produção de uma combinação bastante versátil de produtos, com boa eficiência na transformação dos compostos sólidos em gasosos de maior interesse à indústria química, como $\mathrm{H}_{2}, \mathrm{CO}, \mathrm{CO}_{2}$ e $\mathrm{CH}_{4}$ (MIRANDA, 2014). Além disso, vale destacar a possibilidade de utilização dos resíduos da biomassa no processo de 


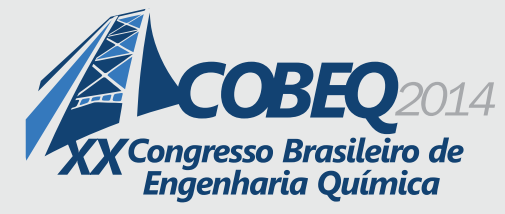

gaseificação, de forma a fornecer uma alternativa ao emprego da biomassa na produção de fertilizantes e até mesmo na produção direta de energia pelas usinas sucro-alcooleiras, em que o preço da energia é mais elevado do que a produzida pelas usinas hidroelétricas.

De acordo com Ahmed et al. (2012), diversos tipos de reatores podem ser empregados para promover a gaseificação da biomassa: reatores de leito fixo ou leito móvel, reatores de leito de arrasto e reatores de leito fluidizado. Dois tipos são mais estudados, a saber: reator de leito borbulhante, com formação de bolhas no leito, e reator de leito circulante, com transporte pneumático das partículas sólidas. Reatores de leito fluidizado são favorecidos para uso neste processo devido, principalmente, ao alto grau de mistura das fases e à consequente eficiente transferência de calor e de massa. Em particular, este trabalho trata da modelagem matemática de um reator de leito borbulhante.

Assim, o presente trabalho tem como principal objetivo desenvolver e implementar um modelo fenomenológico não estacionário para descrever o comportamento do reator em leito fluidizado borbulhante a três fases: emulsão, bolha e sólidos. O modelo proposto inclui três sub-sistemas: a fluidodinâmica, que descreve o comportamento do leito fluidizado; a cinética, que abrange as reações de gaseificação e o consumo da biomassa; e os balanços de massa e energia para as três fases consideradas.

\section{DESENVOLVIMENTO DO MODELO}

A seguir, são apresentados o modelo fluidodinâmico usado nesse trabalho bem como o equacionamento dos balanços de massa e energia no leito borbulhante. As equações que expressam as taxas das reações químicas envolvidas (listadas a seguir) não foram apresentadas aqui, mas esse detalhamento pode ser encontrado em (MIRANDA, 2014).

\subsection{Modelo Fluidodinâmico}

A caracterização fluidodinâmica é baseada no trabalho de Kunii e Levenspiel (1991), levandose em conta os parâmetros apresentados a seguir.

Velocidade de mínima fluidização (BOTTERILL e BESSANT, 1976):

$$
U_{m f}=\left(\frac{\mu_{g}}{\rho_{g} \cdot d_{p}}\right)\left(\sqrt{1135,7+0,0408 \cdot\left(\frac{d_{p}{ }^{3} \cdot \rho_{g} \cdot g\left(\rho_{s}-\rho_{g}\right)}{\mu_{g}^{2}}\right)}-33,7\right)
$$

Diâmetro da bolha (MORI, WEN, 1975):

$$
\begin{aligned}
& d_{b}=d_{b m}-\left(d_{b m}-0,0376 \cdot\left(U-U_{m f}\right)^{2}\right) \exp \left(-\frac{0,3 \cdot z}{D}\right) \\
& \mathrm{d}_{\mathrm{bm}}=0,652 \cdot\left[\mathrm{A}_{\mathrm{t}}\left(\mathrm{U}-\mathrm{U}_{\mathrm{mf}}\right)\right]^{0,4}
\end{aligned}
$$

Velocidade ascendente da bolha (DAVIDSON e HARRISON, 1963): 
$U_{b}=U-U_{m f}+0,711 \sqrt{g \cdot d_{b}}$

Porosidade de mínima fluidização (KAUSHAL et al., 2010):

$$
\varepsilon_{m f}=0,478\left(\frac{d_{p}{ }^{3} \cdot \rho_{g} \cdot g\left(\rho_{s}-\rho_{g}\right)}{\mu_{g}^{2}}\right)^{-0,018}
$$

Transferência de massa entre as fases bolha e emulsão (KUNII e LEVENSPIEL 1991):

$$
\frac{1}{K_{b e}}=\left(4,5 \frac{U_{m f}}{d_{b}}+5,85 \frac{D_{H 2 O}^{0,5} \cdot g^{0,25}}{d_{b}^{1,25}}\right)^{-1}+\left(6,78\left(\frac{\varepsilon_{m f} \cdot D_{H 2 O} \cdot U_{b}}{d_{b}^{3}}\right)^{0,5}\right)^{-1}
$$

Transferência de calor entre as fases bolha e emulsão (KUNII e LEVENSPIEL 1991):

$$
\begin{aligned}
\frac{1}{H_{b e}}= & \left(4,5 \frac{U_{m f} \cdot \rho_{H 2 O} \cdot C_{p H 2 O}}{d_{b}}+5,85 \frac{\left(k_{H 2 O} \cdot \rho_{H 2 O} \cdot C_{p H 2 O}\right)^{0,5} \cdot g^{0,25}}{d_{b}^{1,25}}\right)^{-1} \\
& +\left(6,78\left(\frac{\varepsilon_{m f} \cdot k_{H 2 O} \cdot \rho_{H 2 O} \cdot C_{p H 2 O} \cdot U_{b}}{d_{b}^{3}}\right)^{0,5}\right)^{-1}
\end{aligned}
$$

Transferência de calor entre as fases emulsão e sólida (KUNII e LEVENSPIEL 1991):

$$
H_{s e}=\frac{\left(2+0,6 \cdot \operatorname{Pr}^{\frac{1}{3}} \cdot \operatorname{Re}_{s}^{\frac{1}{2}}\right) \cdot k_{H 2 O}}{d_{p}}
$$

sendo o número de Prandtl (Pr) calculado em função das características do agente gaseificante e o número de Reynolds (Re) calculado em função das características da partícula sólida inserida no meio de fluidização.

\subsection{Modelo Cinético}

A biomassa foi interpretada como sendo um composto de fórmula geral $\mathrm{C}_{\mathrm{x}} \mathrm{H}_{\mathrm{y}} \mathrm{O}_{\mathrm{z}}$. As reações constituintes desse modelo são as seguintes, cujas taxas de reação estão especificadas no trabalho de Corella e Sanz (2005) e Miranda (2014):

- Desvolatilização (R1): $\mathrm{C}_{\mathrm{x}} \mathrm{H}_{\mathrm{y}} \mathrm{O}_{\mathrm{z}} \rightarrow$ Alcatrão1 + Asfalto1 $+\mathrm{H}_{2}+\mathrm{CO}_{2}+\mathrm{CO}+\mathrm{CH}_{4}+\mathrm{C}_{2} \mathrm{H}_{4}$

- Reação de oxidação de alcatrão (R2): Alcatrão1 + 0,680 $\rightarrow 0,31 \mathrm{H}_{2} \mathrm{O}+0,25 \mathrm{CO}_{2}+0,75 \mathrm{CO}$

- Reação de oxidação de asfalto (R3): Asfalto1 $+0,58 \mathrm{O}_{2} \rightarrow 0,073 \mathrm{H}_{2} \mathrm{O}+0,25 \mathrm{CO}_{2}+0,75 \mathrm{CO}$

- Reação de oxidação de hidrogênio (R4): $\mathrm{H}_{2}+0,5 \mathrm{O}_{2} \rightarrow \mathrm{H}_{2} \mathrm{O}$

- Reação de oxidação de monóxido de carbono (R5): $\mathrm{CO}+0,5 \mathrm{O}_{2} \rightarrow \mathrm{CO}_{2}$ 
- Reação de oxidação de metano (R6): $\mathrm{CH}_{4}+2 \mathrm{O}_{2} \rightarrow \mathrm{CO}_{2}+2 \mathrm{H}_{2} \mathrm{O}$

- Reação de oxidação de eteno (R7): $\mathrm{C}_{2} \mathrm{H}_{4}+3 \mathrm{O}_{2} \rightarrow 2 \mathrm{CO}_{2}+2 \mathrm{H}_{2} \mathrm{O}$

- Reação de reforma a vapor de metano (R8): $\mathrm{CH}_{2}+\mathrm{H}_{2} \mathrm{O} \rightarrow \mathrm{CO}+3 \mathrm{H}_{2}$

- Reação de reforma a vapor de alcatrão1 (R9): Alcatrão1 + 0,15 $\mathrm{H}_{2} \mathrm{O} \rightarrow 0,35 \mathrm{CO}+0,13 \mathrm{H}_{2}+$ 1,2Alcatrão2

- Reação de reforma a vapor de asfalto1: Asfalto1 $+0,38 \mathrm{H}_{2} \mathrm{O} \rightarrow 0,54 \mathrm{CO}+0,45 \mathrm{H}_{2}$

- Reação de reforma a seco de asfalto1: Asfalto1 $+\mathrm{CO}_{2} \rightarrow 1,7 \mathrm{CO}+$ Asfalto2

- Reação de deslocamento de água: $\mathrm{CO}+\mathrm{H}_{2} \mathrm{O} \leftrightarrow \mathrm{CO}_{2}+\mathrm{H}_{2}$

Asfalto2 e Alcatrão2 também podem sofrer oxidação de acordo com as reações R2 e R3.

\subsection{Balanços de Massa e de Energia}

O leito fluidizado borbulhante é constituído por uma fase densa, chamada de emulsão, formada por partículas sólidas e gás, e uma fase formada somente por gás, admitida livre de partículas, chamada de bolha. A fase densa emulsão pode ser dividida em fase emulsão, considerando somente o gás, e fase sólida, considerando somente as partículas sólidas. Assim, o estudo do leito em questão é dividido em três fases: bolha (constituída apenas de gás), emulsão (parte do gás que interage com os sólidos) e sólida (partículas de biomassa). Tanto a fase bolha como a fase emulsão são descritas como um sistema distribuído, em que há variação de propriedades somente na direção axial, enquanto que a fase sólida é descrita como um sistema concentrado, devido à fluidização. Admite-se, também, a troca de calor entre as fases bolha e emulsão e emulsão e sólidos, apenas, já que é considerada a ausência de sólidos nas bolhas.

Balanço de massa na bolha:

$$
\frac{\partial C_{i b}}{\partial t}=D_{i b} \frac{\partial{ }^{2} C_{i b}}{\partial z^{2}}-\frac{\partial}{\partial z}\left(C_{i b} \cdot U_{b}\right)+K_{b e}\left(C_{i b}-C_{i e}\right)+\sum \alpha_{i} \cdot r_{k i b}
$$

Balanço de massa na emulsão:

$\frac{\partial C_{i e}}{\partial t}=D_{i e} \frac{\partial^{2} C_{i e}}{\partial z^{2}}-U_{e} \frac{\partial C_{i e}}{\partial z}+K_{b e}\left(C_{i b}-C_{i e}\right)+\sum \alpha_{i} \cdot r_{k i e}$

Balanço de massa nos sólidos:

$$
\frac{d C_{s}}{d t}=\dot{m}_{e}-\dot{m}_{s}-\sum r_{k s}
$$

Balanço de energia na bolha:

$$
\sum \rho_{i} \cdot C_{p i} \frac{\partial T_{b}}{\partial t}=-\sum \rho_{i} \cdot C_{p i} \frac{\partial}{\partial z}\left(U_{b} \cdot T_{b}\right)+H_{b e}\left(T_{e}-T_{b}\right)+\sum r_{k b} \cdot \Delta H_{k}
$$




\section{$\underline{\text { Balanço de energia na emulsão }}$}

$$
\begin{aligned}
\sum \rho_{i} \cdot C_{p i} \frac{\partial T_{e}}{\partial t}= & -U_{m f} \sum \rho_{i} \cdot C_{p i} \frac{\partial T_{e}}{\partial z}+H_{b e}\left(T_{b}-T_{e}\right)+a_{s} \cdot H_{s e}\left(T_{s}-T_{e}\right) \\
& +\sum r_{k e} \cdot \Delta H_{k}
\end{aligned}
$$

\section{$\underline{\text { Balanço de energia nos sólidos }}$}

$$
\rho_{s} \cdot C_{p s} \frac{d T_{s}}{d t}=-\rho_{s} \cdot C_{p s} \cdot U_{m f} \frac{\partial T_{s}}{\partial z}+a_{s} \cdot H_{s e}\left(T_{e}-T_{s}\right)-\sum r_{k s} \cdot \Delta H_{k}
$$

Nos balanços de massa e de energia são utilizadas as condições de contorno de Danckwerts (1953) e a discretização das equações diferenciais parciais obtidas foi realizada pelo método das diferenças finitas utilizando a fórmula da diferença central. Assim, o sistema final é composto de equações algébricas e de equações diferenciais ordinárias. A resolução do sistema foi implementada em Fortran 90, com o uso do integrador DASSL (MIRANDA, 2014).

\section{RESULTADOS E DISCUSSÃO}

Os resultados apresentados aqui estão divididos em duas partes, a saber: i) inicialmente, apresenta-se a validação do modelo desenvolvido para um caso isotérmico e considerando uma biomassa constituída apenas de carbono, uma vez que há, na literatura, dados experimentais disponíveis para tal cenário, e ii) a análise do modelo considerando uma biomassa mais real, sendo esse o objetivo central da presente comunicação. A cinética da gaseificação da biomassa, para o caso i, bem como o detalhamento das simulações, não estão descritos aqui, mas podem ser encontrados em Miranda (2014). Para o caso ii, madeira de pinho foi considerada como biomassa, com fórmula molecular igual a $\mathrm{C}_{4,2} \mathrm{H}_{5,8} \mathrm{O}_{2,8}$, sendo alimentada a uma vazão de $3,9 \cdot 10^{-4} \mathrm{~kg} / \mathrm{s}$. Desta maneira, a fórmula química para o Alcatrão1 é $\mathrm{CH}_{0,85} \mathrm{O}_{0,17}$, para o Asfalto1 é $\mathrm{CH}_{0,2} \mathrm{O}_{0,13}$, para o Alcatrão2 é $\mathrm{C}_{0,54} \mathrm{H}_{0,54}$ e para o Asfalto2 é $\mathrm{C}_{0,3} \mathrm{H}_{0,15} \mathrm{O}_{0,46}$ (CORELLA, SANZ, 2005). As características intrínsecas das partículas foram mantidas constantes na simulação e iguais àquelas consideradas por Raman et al. (1981), bem como os parâmetros admitidos neste trabalho. Admitiu-se, ainda, que as condições iniciais são iguais às condições de alimentação com injeção somente de água $\left(8,2 \cdot 10^{-3} \mathrm{kmol} / \mathrm{s}\right)$ e oxigênio $\left(1,64 \cdot 10^{-2} \mathrm{kmol} / \mathrm{s}\right)$.

Na Tabela 1, uma comparação é feita entre os dados obtidos pelo modelo deste trabalho com os dados experimentais apresentados no trabalho de Raman et al. (1981), usando biomassa como carbono puro, nas mesmas condições experimentais utilizadas pelo autor. Pode-se observar que os valores calculados pelo modelo parecem se ajustar bem aos dados experimentais. Porém, há desvios evidentes para $\mathrm{CO}_{2}$ e $\mathrm{H}_{2}$, o que pode estar associado à não inclusão de todas as reações que podem ocorrer no processo de gaseificação, decorrente da consideração da biomassa como carbono puro, pois a biomassa real apresenta quantidades consideráveis de oxigênio e hidrogênio, explicando, assim, os menores valores experimentais de $\mathrm{CO}_{2}$ e os maiores valores experimentais de $\mathrm{H}_{2}$ obtidos por 


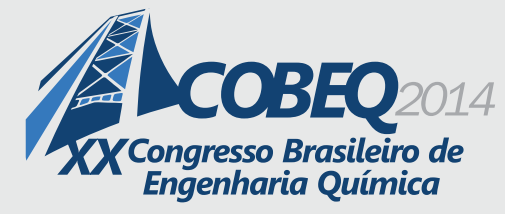

Raman et al. (1981).

Tabela 1 - Validação do modelo com resultados experimentais de Raman et al. (1981)

\begin{tabular}{|c|c|c|}
\hline Componente & $\begin{array}{c}\text { Dados experimentais } \\
\text { (RAMAN et al., 1981) }\end{array}$ & $\begin{array}{c}\text { Dados calculados } \\
\text { (MIRANDA, 2014) }\end{array}$ \\
\hline $\mathrm{CO}$ & 19,30 & 22,97 \\
\hline $\mathrm{H}_{2}$ & 36,88 & 19,94 \\
\hline $\mathrm{CO}_{2}$ & 37,03 & 53,56 \\
\hline $\mathrm{CH}_{4}$ & 6,78 & 3,53 \\
\hline
\end{tabular}

Após a validação do modelo fluidodinâmico e dos balanços de massa e energia desenvolvidos, procedeu-se com a análise do caso considerando uma biomassa mais real. Inicialmente, uma simulação foi realizada considerando temperatura constante de $850{ }^{\circ} \mathrm{C}$, como mostra a Figura 1 . Analisando-se a Figura 1, pode-se perceber que há elevada produção de $\mathrm{H}_{2}$ e $\mathrm{CO}$ com $\mathrm{CH}_{4}$ sendo praticamente todo convertido em $\mathrm{CO}$ e $\mathrm{CO}_{2}$. Vale destacar ainda a formação de água que pode ser explicada pela consideração de reações de oxidação completa ou total, em que há a formação de $\mathrm{CO}_{2}$ e $\mathrm{H}_{2} \mathrm{O}$, ao invés de produtos da combustão incompleta, $\mathrm{H}_{2}$ e CO. Além disso, foram obtidos, em base seca, $33,6 \%$ de $\mathrm{CO}, 33,5 \%$ de $\mathrm{CO}_{2}, 31,9 \%$ de $\mathrm{H}_{2}, 1,0 \%$ de $\mathrm{C}_{2} \mathrm{H}_{4}$ e $0 \%$ de $\mathrm{CH}_{4}$.

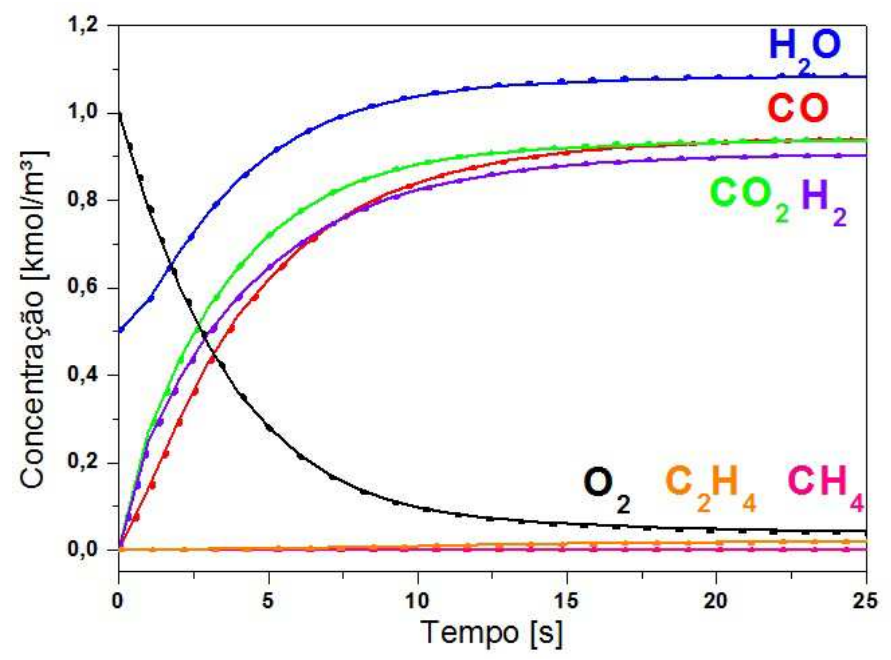

Figura 1: Perfis de concentração dos produtos na saída do reator considerando temperatura constante.

O efeito da variação da temperatura pode ser observado na Figura 2. Comparando-se a Figura 1 com a Figura 2a, é possível perceber que há um aumento na produção de $\mathrm{CO}$, principalmente, e de $\mathrm{H}_{2}$ (gases que constituem o gás de síntese), causados pela variação da temperatura. Explicitando numericamente, foram obtidos, em base seca, 56,3\% de $\mathrm{CO}, 14,1 \%$ de $\mathrm{CO}_{2}, 27,9 \%$ de $\mathrm{H}_{2}, 1,3 \%$ de $\mathrm{C}_{2} \mathrm{H}_{4}$ e $0,4 \%$ de $\mathrm{CH}_{4}$. Na Figura $2 \mathrm{~b}$, a temperatura aumenta gradativamente devido somente à troca de calor entre as fases. Ao atingir a temperatura de reação de desvolatilização $\left(\sim 330^{\circ} \mathrm{C}\right)$, o sistema reacional efetivamente se inicia e o aumento abrupto da temperatura, com posterior queda e estabilização, se deve, respectivamente, à ocorrência de reações de oxidação e à ocorrência das 


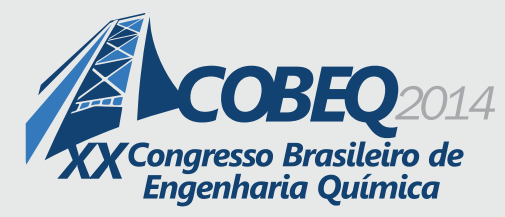

reações de gaseificação. Esse comportamento ilustra a dinâmica particular do processo conduzido com biomassa real, podendo a operação e o controle do reator exigir atenção especial.

Na Figura 2a, observa-se, ainda, que em torno de 30min (2.000s) ocorre um pico de formação de $\mathrm{H}_{2}$, com ligeira diminuição na quantidade de $\mathrm{CO}$, aumento de $\mathrm{CO}_{2} \mathrm{e}$, principalmente, maior consumo de $\mathrm{H}_{2} \mathrm{O}$. De acordo com a Figura $2 b$, isso ocorre por causa da temperatura elevada alcançada pelo sistema, em torno de $840{ }^{\circ} \mathrm{C}$, beneficiando o processo de gaseificação.
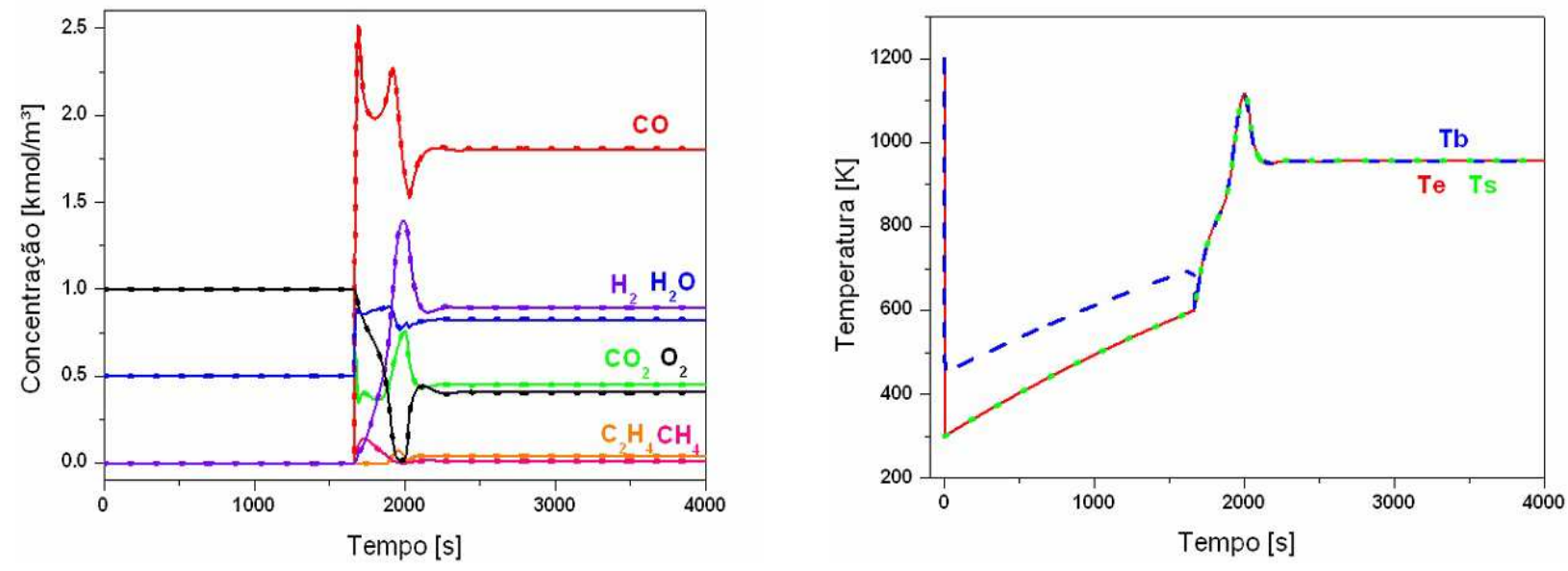

Figura 2: Perfis de (a) concentração dos produtos e de (b) temperatura na saída do reator considerando variação de temperatura.

\section{CONCLUSÕES}

Neste trabalho, um modelo fluidodinâmico para o reator de leito fluidizado a três fases foi proposto. A análise de simulações conduzidas com uma biomassa com características reais mostrou a adequação do modelo cinético empregado, já que diversos trabalhos na literatura consideram a biomassa composta apenas por carbono (RAMAN, 1981; GÓMEZ-BAREA, LECKNER, 2010; GORDILLO, BELGHIT, 2010). Os resultados obtidos mostram que uma biomassa real pode induzir comportamento dinâmico e complexo do reator quando a variação da temperatura é avaliada, modificando expressivamente as concentrações de produtos na saída.

\section{NOMENCLATURA}

$\begin{array}{llllll}\beta-\mathrm{kg} & \mathrm{a}_{\mathrm{s}}-\mathrm{m}^{2} / \mathrm{m}^{3} & \mathrm{~d}_{\mathrm{b}}-\mathrm{m} & \mathrm{H}_{\mathrm{be}}-\mathrm{kJ} / \mathrm{m}^{3} / \mathrm{K} / \mathrm{s} & \dot{\mathrm{m}}_{\mathrm{s}}-\mathrm{kg} / \mathrm{s} & \mathrm{U}-\mathrm{m} / \mathrm{s} \\ \Delta \mathrm{H}-\mathrm{kJ} / \mathrm{kmol} & \mathrm{A}_{\mathrm{t}}-\mathrm{m}^{2} & \mathrm{~d}_{\mathrm{p}}-\mathrm{m} & \mathrm{H}_{\mathrm{se}}-\mathrm{kJ} / \mathrm{m}^{2} / \mathrm{K} / \mathrm{s} & \mathrm{r}-\mathrm{kmol} / \mathrm{m}^{3} / \mathrm{s} & \mathrm{U}_{\mathrm{b}}-\mathrm{m} / \mathrm{s} \\ \varepsilon_{\mathrm{mf}}-\mathrm{adm} & \mathrm{C}-\mathrm{kmol} / \mathrm{m}^{3} & \mathrm{D}-\mathrm{m} & \mathrm{k}-\mathrm{kJ} / \mathrm{m} / \mathrm{K} / \mathrm{s} & \mathrm{T}-\mathrm{K} & \mathrm{U}_{\mathrm{m}}-\mathrm{m} / \mathrm{s} \\ \mu-\mathrm{Pa} . \mathrm{s} & \mathrm{C} p_{\mathrm{i}}-\mathrm{kJ} / \mathrm{kmol} / \mathrm{K} & \mathrm{D}_{\mathrm{i}}-\mathrm{m}^{2} / \mathrm{s} & \mathrm{K}_{\mathrm{be}}-1 / \mathrm{s} & \mathrm{t}-\mathrm{s} & \mathrm{Z}-\mathrm{m} \\ \rho-\mathrm{kg} / \mathrm{m}^{3} & \mathrm{C} \mathrm{p}_{\mathrm{s}}-\mathrm{kJ} / \mathrm{kg} / \mathrm{K} & \mathrm{g}-\mathrm{m} / \mathrm{s}^{2} & \dot{\mathrm{m}}_{\mathrm{e}}-\mathrm{kg} / \mathrm{s} & & \end{array}$

Subscrito: 
b-bolha $\quad \mathrm{i}$ - componente $\left(\mathrm{H}_{2}, \mathrm{CO}, \mathrm{CO}_{2}, \mathrm{CH}_{4}, \mathrm{C}_{2} \mathrm{H}_{4}, \mathrm{O}_{2}, \mathrm{H}_{2} \mathrm{O}\right)$

e-emulsão $\mathrm{g}$-agente gaseificante $\mathrm{mf}$ - mínima fluidização $\mathrm{s}$ - sólidos

\section{REFERÊNCIAS}

AHMED, T.Y.; AHMAD, M.M.; YUSUP, S.; INAYAT, A.; KHAN, Z. Mathematical and computacional approaches for design of biomass gasification for hydrogen production: a review. Ren. Sust. Energy Rev., v. 16, p. 2304-2315, 2012.

BASU, P. Biomass gasification and pyrolysis - Practical Design. MA: Ed. Academic Press. Burlington, 2010.

BOTTERILL, J.S.; BESSANT, D.J. The flow properties of fluidized solids. Powder Technol., v.14, n.1, p.131-137, 1976.

CORELLA, J.; SANZ, A. Modeling circulating fluidized bed biomass gasifiers. A pseudo-rigorous model for stationary state. Fuel Process. Technol., v. 86, p. 1021-1053, 2005.

DANCKWERTS, P.V. Continuous flow systems. Distribution of residence times. Chem. Eng. Sci., v.1, p. 1-13, 1953.

DAVIDSON, J.F.; HARRISON, D. Fluidised Particles. New York: Cambridge University Press, 1963.

GÓMEZ-BAREA, A.; LECKNER, B. Modeling of biomass gasification in fluidized bed. Prog. Energy Combust. Sci., v. 36, n. 4, p. 444-509, 2010.

GORDILLO, E. D.; BELGHIT, A. A two phase model of high temperature steam-only gasification of biomass char in bubbling fluidized bed reactors using nuclear heat. Int. J. Hydrogen Energy, v. 36, n. 1, p. 374-381, 2010.

KAUSHAL, P.; ABEDI, J.; MAHINPEY, N. A comprehensive mathematical model for biomass gasification in a bubbling fluidized bed reactor. Fuel, v. 89, p. 3650-3661, 2010.

KUNII, D.; LEVENSPIEL, O. Fluidization Engineering. MA: Ed. Butterworth-Heinemann. Newton, 1991.

MIRANDA, Gilliani Peixoto. Modelagem e Simulação de Reatores de Leito Fluidizado para a Gaseificação da Biomassa. Rio de Janeiro, 2014. 110 folhas. Dissertação (Mestrado em Engenharia Química) - Programa de Pós-graduação em Engenharia Química, COPPE, Universidade Federal do Rio de Janeiro, Rio de Janeiro, 2014.

MORI, S.; WEN, C.Y. Estimation of bubble diameter in gaseous fluidized beds. AIChE J., v.21, p.109-115, 1975.

RAMAN, P.; WALAWENDER, W. P.; FAN, L. T.; et al. Mathematical Model for the Fluid-Bed Gasitication of Biomass Materials. Application. I\&EC Proc. Des. Dev., v. 20, p. 686-692, 1981.

SADAKA, S. S.; GHALY, A. E.; SABBAH, M. A. Two phase biomass air-steam gasification model for fuidized bed reactors: Part I — model development. Biomass Bioenergy, v. 22, p. 439-462, 2002. 